\title{
Combinatorial Auction-based Multiple Dynamic Mission Assignment
}

\author{
Jin-Hee Cho, Ananthram Swami, and Trevor Cook \\ U.S. Army Research Laboratory \\ Computational and Information Sciences Directorate \\ Adelphi, MD 20783 \\ \{jinhee.cho, ananthram.swami, trevor.j.cook\}.civ@mail.mil
}

\begin{abstract}
This paper proposes a combinatorial auction based approach to solving a multiple dynamic mission assignment (MDMA) problem. The problem presents itself in scenarios in which mobile nodes perform multiple missions, and the missions are supported by multiple nodes in a mobile ad hoc network. In this scenario, missions may dynamically arrive, may have to be completed at different times, and different numbers of members may be required for mission execution. The proposed MDMA protocol aims to minimize communication overhead caused by the mission assignment while maximizing each node's utilization (i.e., minimizing its idle time) and maximizing the fraction of mission completion over the initial missions given. We compare the proposed MDMA protocol with baseline mechanisms such as a single-item auction or a single-minded auction based on the above performance metrics.
\end{abstract}

Keywords-combinatorial auction, single-item auction, task assignment, dynamic mission, mobile ad hoc networks, auction bundle algorithm, single-minded auction

\section{INTRODUCTION}

Tactical networks often have dynamic missions assigned for execution by multiple members where the missions arrive at different times and have to be completed within given, possibly different deadlines. For example, in the theatre of military operations, multiple missions may dynamically arise that require unmanned aerial vehicles (UAVs) or (static/mobile) sensors or other assets, and since they are a constrained resource, we must decide how best to allocate these assets to the missions to best support the army's goals. Furthermore, we must perform the allocations in an efficient way, so as to reliably deliver high quality services with minimum overhead.

This work proposes a multiple mission assignment mechanism based on combinatorial auction theory for tactical mobile ad hoc networks (MANETs). Each node will execute multiple missions during its lifetime and each mission may be performed by multiple members. In this tactical network, each node has a goal of maximizing its utilization by reducing idle time. On the other hand, in terms of the mission leaders' perspective, missions should be optimally assigned to maximize successful mission completion.

The task assignment problem has been well studied in the areas of UAVs, robot-to-robot networks, and sensor-mission (or task) matching in wireless sensor networks (WSNs) [1], [6], [7], [10]. A market-based approach to model dynamic, distributed resource allocation problems was proposed in [11]. The underlying idea is that market-based mechanisms are able to facilitate decentralized resource allocation in terms of complex tradeoffs of goods and services in sensor networks just as in human societies [11]. Mainland et al. [10] used an auction theory to propose a decentralized resource allocation algorithm in sensor networks. Choi et al. [6] proposed consensus-based algorithms to coordinate a fleet of mobile autonomous robots. Ahmed et al. [1] proposed a dynamic auctioning scheme for UAV search and rescue mission. However, all these works [1], [6], [10] only considered static missions (i.e., all missions are given, with the same timeframe of arrival and completion) and each node performs only one mission or task during its entire lifetime. In our formulation, a node may perform multiple missions.

Dynamic auction-based resource allocation schemes are also used in cloud computing [2], [8]. Lin et al. [8] proposed a dynamic auction mechanism maximizing profit of the cloud service provider via efficient allocation of its computational resources. An et al. [2] studied resource allocation algorithms to maximize the utility of service providers in cloud computing. Both works assume a static setting of service providers and customers. However, we consider the dynamics of node failure or disconnection/reconnection in this work.

The performance of auction algorithms has been investigated via metrics such as delay or network lifetime [12], [13]. Park et al. [12] used a delay metric that combines the overall computation time plus coordination delay. Tei et al. [13] proposed an auction-based route allocation approach to maximize network lifetime in terms of energy consumption in MANETs. Different from [12], [13], our work uses the metrics of communication overhead, a node's resource utilization (i.e., busy time) and the fraction of completed missions in order to reflect relevant performance aspects of military network environments.

Recently market-based approaches were proposed [7] to model dynamic missions or a mission-asset matching problem, which seems similar to this work. Johnson et al. [7] studied a multiple mission assignment problem for wireless stationary sensor networks where missions are modeled as static (i.e., all missions start and finish at the same time) or dynamic (i.e., missions arrive and should be completed by different times). For static missions, they also used combinatorial auction theory, modeling sensors as bid items and missions as bidders. In the 
dynamic mission setting, an "arrived" mission broadcasts its arrival to look for qualified local sensor nodes. In both cases, each node can be assigned to only one mission during its lifetime while one mission requires participation of multiple nodes. Since nodes are stationary, only local nodes can be assigned to a mission. Our work differs from the approach in [7] in that we deal with dynamic missions in MANETs where each mission requires multiple nodes' participation, while each node also may need to execute multiple missions during its lifetime. Because of the MANET environment, asset allocation is no longer strictly local.

In this work, we adopt a combinatorial auction algorithm (CAA) [3] to solve a dynamic multiple mission assignment problem. In combinatorial auctions, a large number of items are auctioned simultaneously and bidders are allowed to express their preferences over available bundles of items. Auctioneers inform winners based on the bid prices. The literature discusses the main advantages of using combinatorial auctions [3]. First, CAA significantly reduces the amount of communications between auctioneers and bidders (information regarding items or bidders' preferences, or winner information). Second, CAA reveals less private information (only showing information on a bundle, not all the details for each item), resulting in less information sharing and potentially increased security. We mainly exploit the first advantage of CAA in this work.

The main contributions of this work are as follows. First, this work is the first to deal with a multiple dynamic mission assignment (MDMA) problem where each mobile node may need to perform multiple missions during its entire lifetime without being restricted by fixed locations of mission leaders or missions. Second, this work is the first that employs CAA for MDMA, aiming at reducing communication overhead of the proposed auction protocol while maximizing system goals of mission completion and each node's utilization. Third, this work first studies the performance of the proposed CAA-based resource allocation scheme under dynamic network conditions, where the dynamics of nodes joining and leaving the network leads to reassignment of tasks. Last, this work also compares the proposed scheme against baseline models such as a singleauction (i.e., auction one item at a time) or a single-minded auction (i.e., a bidder insists on only one preference over multiple bundles available in the bid).

The rest of this paper is organized as follows. Models and assumptions are discussed in Section 2; an overview of the proposed scheme and performance metrics is provided in Section 3; simulation results and physical interpretations are discussed in Section 4; and additional discussion is provided in Section 5.

\section{SYSTEM MODEL}

\section{A. Network Model}

We consider a tactical MANET environment where nodes communicate with each other through multiple hops. Heterogeneous MANET nodes are homogeneously spread out in a square-shaped operational area with $\mathrm{m} \times \mathrm{m}$ regions where no malicious nodes are present. Participating nodes may move with different speeds, reflecting the typical heterogeneity in a MANET. Further, each node's capacity to disseminate packets per second is limited by its "average workload." We adopt a hierarchical network structure [5] for scalability by having a leader in each region, called "regional leader". For any communications between two nodes in two different regions, including communications for auctioning, a packet is disseminated through the hierarchical structure such as noderegional leader - regional leader - node. This is taken into consideration for computing communication overhead in our performance metric. A mobile node may be involuntarily disconnected or reconnected due to terrain or unreliable wireless transmission. Also a node may voluntarily leave or join the network for tactical reasons. These are all implicitly considered as node leave and join events where the intervals between departures and rejoins are modeled as exponentially distributed with rates $\lambda$ and $\mu$.

Each node may require some amount of time before it is available to support a mission. For instance, nodes may need time to move towards a location where they have a suitable view to support a mission. We model this by requiring supporting nodes to be in the same region as mission leaders. So, as soon as a node is assigned to a mission, it will move towards the mission leader's location. Whenever a node should switch from one mission to another, it moves towards the location of the next mission leader. While a node is executing a mission, it will move around within the region the mission leader resides. Since participating nodes may move with different speeds and inter-mission distances may be significant, the effect of switching missions becomes an important aspect which is accounted for in this MDMA model.

Multiple missions are given to mission leaders where each mission requires a certain number of member nodes for execution. As soon as a node is assigned to a mission, it will move towards the mission leader's location. When a node is assigned to multiple missions, whenever it should switch from one mission to another, it moves towards the location of the next mission leader. A mission leader is selected based on the capacity of the average workload, called "super-node." The mission leaders are selected as soon as missions are defined, and before the auction phase. A regional leader is randomly selected and reelected when it moves to another region. A regional leader may be selected as a mission leader but not necessarily.

We model missions as items and member nodes as bidders. Thus, each bundle consists of multiple missions and the available bundles will be determined based on the auction bundle algorithm discussed in Section II.C. Each node may express multiple preferences in its bid to maximize its chances of winning a bid. We assume that nodes do not behave maliciously such as lying about preferences or valuations of preferred bundles in the auction process.

\section{B. Mission Model}

In our model, dynamic missions may arrive (or start at) and have to be completed by different times. We assume that all missions are to be completed within the entire session time, $\mathrm{T}_{\text {session. }}$. As an example of dynamic missions, Fig. 1 shows the arrival (start) times and completion deadlines for 20 different missions. 


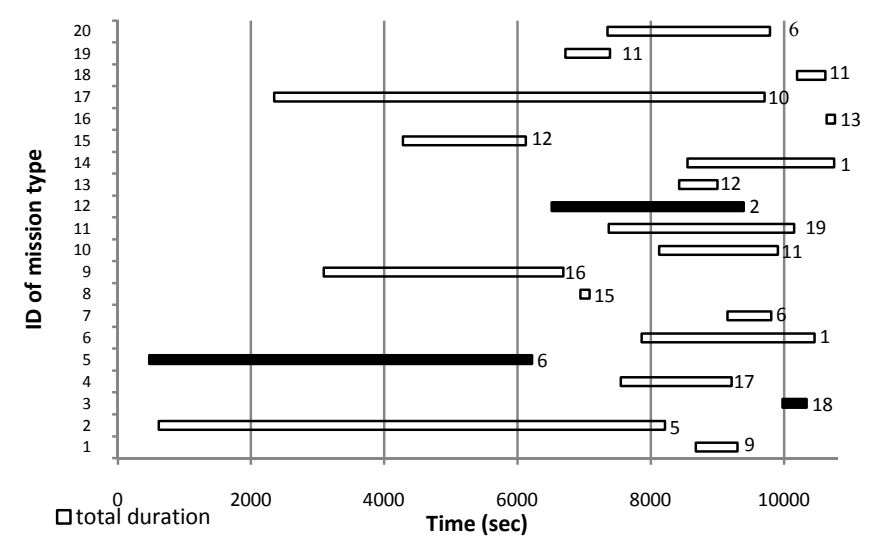

Fig. 1: Dynamic mission duration with different arrival and completion times.

Each bar indicates the duration of a mission; and each mission requires a certain number of members for mission completion, as labeled in Fig. 1. Also, each mission has a different workload for its completion (i.e., the number of packets to disseminate per second). The proposed scheme allocates multiple missions into one bundle without any schedule conflicts. For example, mission IDs (see y-axis) 5, 12, and 3 (indicated in black in Fig. 1) can be in one bundle, i.e., a node can sequentially perform the three missions. The next section will discuss the auction bundle algorithm in detail.

\section{Dynamic Multiple Mission Assignment}

We employ combinatorial auctions for efficient resource allocation. In this work, nodes are bidders, mission leaders are auctioneers, and items to sell are bundles consisting of multiple missions. After mission leaders agree on bundling items to sell, those bundles are informed to potential bidders. Bidders submit their bids to auctioneers with the score(s) of the chosen (preferred) bundle(s). The bid with the highest score is selected as a winner. Each bidder can only win one bundle but can be assigned to multiple missions since one bundle may include multiple missions. We also allow bidders to express multiple preferences over available bundles.

Auction Bundle Algorithm: A bundle consists of multiple missions that can be executed sequentially. Since each mission requires the participation of multiple members, there may exist multiple identical bundles with the same set of missions. Scheduling multiple missions into one bundle with minimum idle time between missions has high computational complexity (e.g., job-shop problem in [4]). To relax it, we bound the maximum number of items (missions) in one bundle, $\mathrm{N}_{\text {item }}^{\max }$. For example, suppose missions $\mathrm{M}_{5}, \mathrm{M}_{12}$, and $\mathrm{M}_{3}$ require 6,2 , and 18 members respectively. Given $N_{\text {item }}^{\max }=3$ and assuming that all three missions are not overlapped in their schedules, there may exist at least 2 bundles with the same components such as the bundle with $\left\{\mathrm{M}_{5}, \mathrm{M}_{12}\right.$, and $\left.\mathrm{M}_{3}\right\}$. When $\mathrm{N}_{\mathrm{item}}^{\max }=1$, it represents a single-item auction as a baseline model. This work uses a greedy algorithm: a bundle is assigned a mission with the minimum waiting time. The description of each bundle includes its required workload $\left(\mathrm{B}_{\mathrm{j}}^{\text {workload }}\right)$ and the sum of durations of composed missions in bundle $\mathrm{j}\left(\mathrm{T}_{\mathrm{j}}^{\text {busy }}\right.$ ) based on specifications (i.e., workload, start/end time) of the missions.

Each mission leader informs its mission's specifications to all other mission leaders during the network deployment period. Each mission leader runs the above heuristic greedy algorithm to generate bundles. This bundle generation is a one-time operation during the network deployment period. However, the composed missions of the initially generated bundles may change during winner determination period or re-allocation of bundles due to nodes' mobility: there may be some deadline adjustment due to lack of members to achieve mission completion or elimination of certain missions from the bundles due to the lack of winner members. If a mission leader fails to collect the required number of members, the mission is regarded as incomplete. Whenever any bundles are available in the auction market, either because they were not assigned in previous auctions or because they need to be reassigned as nodes leave the network, the available bundle information is disseminated to regional leaders that can then broadcast it to their members.

Bid: Each node is a bidder and submits its bid based on the scores of available bundles [3]. Node i's score of bundle $j$ is computed based on its valuation and price by:

$$
\mathrm{C}_{\mathrm{i}, \mathrm{j}}=\mathrm{v}_{\mathrm{j}}-\mathrm{p}_{\mathrm{i}, \mathrm{j}}
$$

The score $\mathrm{C}_{\mathrm{i}, \mathrm{j}}$ indicates the net benefit that node $\mathrm{i}$ gains by bidding on bundle $\mathrm{j}$. Higher scores are desirable. The valuation of bundle $\mathrm{j}, \mathrm{v}_{\mathrm{j}}$, is computed by:

$$
v_{j}=\frac{T_{j}^{\text {busy }}}{T_{\text {session }}}, \quad T_{j}^{\text {busy }} \leq T_{\text {session }}
$$

$\mathrm{T}_{\mathrm{j}}^{\text {busy }}$ is bundle $\mathrm{j}$ 's busy time, the sum of durations of missions in bundle $\mathrm{j}$, and $\mathrm{T}_{\text {session }}$ is the whole session time. This reflects each node's goal of maximizing its utilization (busy period). Notice that $v_{j}$ is the same to all nodes. $p_{i, j}$ is the price node $i$ is willing to pay to take bundle $j$ and is computed by:

$$
\mathrm{p}_{\mathrm{i}, \mathrm{j}}=1-\frac{\mathrm{N}_{\mathrm{i}}^{\text {average-workload }}}{\mathrm{B}_{\mathrm{j}}^{\text {workload }}}
$$

$B_{j}^{\text {workload }}$ is the overall average workload (the average number of packets to send per second) required by bundle $\mathrm{j}$. $\mathrm{N}_{\mathrm{i}}^{\text {average-workload }}$ is the average workload that node $\mathrm{i}$ can handle. $p_{i, j}$ is different per node and can be negative if the workload required by bundle $\mathrm{j}$ is too light compared to its average workload capacity, leading to a higher score for bundle $\mathrm{j}$, as shown in Equation 1. Note that positive prices indicate that the node is overloaded compared to its average workload, and negative prices indicate that the node's average workload is not exceeded.

Each node selects a maximum of $\mathrm{n}$ preferences $\left(\mathrm{N}_{\text {preference }}^{\max }\right)$ over available bundles based on their scores (See Equation 1) and submits the bids (bundle ID and its score) to an auctioneer, the closest mission leader. But each node limits its choices to 
bundles with positive scores, implying that if the bundle requires too much workload, a node will give up bidding.

Winner Determination: The received bids are exchanged among other mission leaders through the network structure. Upon receiving all bids, each mission leader determines winners based on the received bid scores. Recall that there exist bundles with the same mission components. We regard them as a same type bundle. When multiple bidders bid on a same bundle, auctioneers determine winners based on the scores of their bids regardless of the preference rank. When a mission leader does not have sufficient members to execute the mission, mission leaders will check if the mission's deadline (completion time) can be extended based on the available winners (fewer members that are announced as winners) without any schedule conflict among all bundles with the mission. If any conflict occurs, the mission is permanently removed from all bundles. If the delay introduced by the fewer members under the mission is tolerable by having sufficient waiting time to trigger the next mission in all the bundles, the mission is retained only with the extended completion time.

Dynamic Auctions upon Node Leave: We model the dynamics as a certain number of nodes are scheduled to leave and join the network randomly where their inter-arrival time is exponentially distributed. When a node is selected as a winner but leaves the network after the session starts, the bundle returned from the leaving node is auctioned to other potential bidders: current members with no assigned bundles. The current members bid on the available bundles based on the scores of the bundles only with the remaining missions. If mission leaders cannot find the winner of a bundle, the bundle is re-auctioned in a subsequent round. We use a fixed time interval ( $\mathrm{T}_{\text {next }}^{\text {auction }}$ ) for a next round auction. When deciding whether the bundle can be re-auctioned or not in the next round, mission leaders check if the delay incurred due to the leaving node is tolerable by extending the completion time, as discussed earlier. When the bundle cannot be re-auctioned in the next round due to a schedule conflict in all bundles with the mission being currently executed, the current mission is halted and permanently removed from all the bundles with the mission. The removed mission is recorded as incomplete.

\section{PERFORMANCE EVALUATION}

\section{A. Dynamic Combinatorial Auction Process}

The combinatorial auction process of the proposed MDMA protocol is summarized as follows. During network deployment, mission leaders (auctioneers) generate bundles, and select winners through several rounds of auctions. In each round, auctioneers determine winners and check if all members are assigned to bundles or if the previous auction result is the same as the current auction result. If this is the case, the auction stops, and the session starts to execute missions. During the session, some nodes may leave and rejoin the network. Any bundles involving leaving nodes will be auctioned to other potential bidders. The same conditions are also checked for the bundle to be re-auctioned if it is not sold in the previous auction round.

\section{B. Performance Metrics}

This section defines the three performance metrics used in this work: overall utilization, mission completion ratio, and total communication overhead.

Overall Utilization (U): This is the average busy time of all nodes in the system and is computed by:

$$
\mathrm{U}=\frac{\sum_{\mathrm{i} \in \mathrm{I}} \mathrm{U}_{\mathrm{i}}}{|\mathrm{I}|}, \mathrm{U}_{\mathrm{i}}=\frac{\mathrm{T}_{\mathrm{i}}^{\text {busy }}}{\mathrm{T}_{\text {session }}}, \mathrm{T}_{\mathrm{i}}^{\text {busy }} \leq \mathrm{T}_{\text {session }}
$$

I is the set of all initial members in the system and $|\mathrm{I}|$ is the cardinality of the set $I_{\text {. }} T_{i}^{\text {busy }}$ is the busy time of node $i$ and $\mathrm{T}_{\text {session }}$ is the session time within which all missions should be completed. Higher $U$ is desirable to maximize overall node utilization.

Mission Completion Ratio (M): This is the fraction of mission completion weighted by the required number of members per mission during the session and is defined by:

$$
\mathrm{M}=\frac{\sum_{\mathrm{j} \in \mathrm{S}} \mathrm{N}_{\mathrm{j}}^{\text {mission }}}{\sum_{\mathrm{i} \in \mathrm{G}} \mathrm{N}_{\mathrm{i}}^{\text {mission }}}
$$

$\mathrm{S}$ is the set of completed missions and $\mathrm{N}_{\mathrm{j}}^{\text {mission }}$ is the number of completed mission items in mission $\mathrm{j}$. $\mathrm{G}$ is the set of initially assigned missions to the network, and $\mathrm{N}_{\mathrm{i}}^{\text {mission }}$ is the number of nodes required for the execution of mission i. Higher $M$ is desirable to achieve high mission completion.

Communication Overhead ( $\mathbf{C}_{\text {total }}$ ): This is the total communication cost incurred by the proposed scheme. This cost includes the cost for generating bundles among mission leaders $\left(\mathrm{C}_{\text {bundle }}^{\mathrm{ND}}\right)$, disseminating bid information from nodes to mission leaders and winner information from mission leaders to winner nodes during network deployment $\left(\mathrm{C}_{\text {bid }}^{\mathrm{ND}}+\mathrm{C}_{\text {winner }}^{\mathrm{ND}}\right)$ and session $\left(\mathrm{C}_{\text {bid }}^{\text {session }}+\mathrm{C}_{\text {winner }}^{\text {session }}\right)$ respectively and is computed by:

$$
\mathrm{C}_{\text {total }}=\mathrm{C}_{\text {bundle }}^{\mathrm{ND}}+\mathrm{C}_{\mathrm{bid}}^{\mathrm{ND}}+\mathrm{C}_{\text {winner }}^{\mathrm{ND}}+\mathrm{C}_{\text {bid }}^{\text {session }}+\mathrm{C}_{\text {winner }}^{\text {session }}
$$

Each cost is computed based on hop bits unit to reflect multihop communications in MANETs. The cost for generating bundles $\left(\mathrm{C}_{\text {bundle }}^{\mathrm{ND}}\right)$ includes the exchanges of mission information among mission leaders and the dissemination of agreed bundle information to all members in the network as shown by:

$\mathrm{C}_{\text {bundle }}^{\mathrm{ND}}=\sum_{\mathrm{k} \in \mathrm{G}} \mathrm{M}_{\mathrm{k}}^{\text {mission }} \mathrm{N}_{\mathrm{k}-\text { mission }}^{\text {hop }}+\sum_{\mathrm{j} \in \mathrm{B}} \mathrm{M}_{\mathrm{j} \text {-bundle }} \mathrm{N}_{\mathrm{j} \text {-bundle }}^{\text {hop }}$

$\mathrm{G}$ is the set of missions, $\mathrm{M}_{\mathrm{k}}^{\text {mission }}$ is the message size (bits) of mission specification, and $\mathrm{N}_{\mathrm{k}-\text { mission }}^{\text {hop }}$ is the number of hops that the message $\mathrm{M}_{\mathrm{k}}^{\text {mission }}$ travels to reach all members and can be different depending on the locations of mission leaders. $\mathrm{B}$ is the

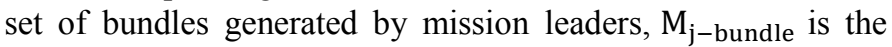
message size (bits) of bundle $\mathrm{j}$ which may be different depending on how many missions are included. $\mathrm{N}_{\mathrm{j} \text {-bundle }}^{\text {hop }}$ is the

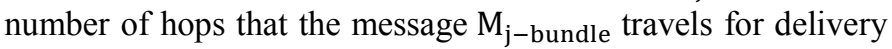
to all members and also can be different depending on the locations of mission leaders. $\mathrm{C}_{\text {bid }}^{\mathrm{ND}}$ is the cost for each bidder to deliver its bid(s) to mission leaders and computed by: 


$$
\mathrm{C}_{\text {bid }}^{\mathrm{ND}}=\sum_{\mathrm{r} \in \mathrm{R}}\left(\sum_{\mathrm{i} \in \mathrm{I}} \mathrm{M}_{\mathrm{i} \text {-bid-r }} \mathrm{N}_{\mathrm{i} \text {-bid-r }}^{\mathrm{hop}}\right)
$$

I is the set of all member nodes in the network, $M_{i-b i d-r}$ is the message size (bits) of the bid(s) that bidder i submits in the $\mathrm{r}^{\text {th }}$ round of auctions and can be different depending on which bundles bidder $\mathrm{i}$ chose and how many bundles it prefers. $\mathrm{N}_{\mathrm{i}-\text { bid-r }}^{\text {hop }}$ is the number of hops the message $\mathrm{M}_{\mathrm{i}-\text { bid-r }}$ travels in the $r^{\text {th }}$ round of auctions until it is received by mission leaders. $\mathrm{R}$ is the set of all auction rounds that occur during network deployment. Since the auction will continue until two conditions (whether all nodes are assigned to bidding bundles or the result of the previous auction round is the same as that of the current auction round) are met, $\mathrm{C}_{\mathrm{bid}}^{\mathrm{ND}}$ accumulates communication costs for all auction rounds during network deployment. After mission leaders decide winners, the winner information is disseminated to all winners with the following cost:

$$
\mathrm{C}_{\text {winner }}^{\mathrm{ND}}=\sum_{\mathrm{r} \in \mathrm{R}} \mathrm{M}_{\text {bundles-r }} \mathrm{N}_{\text {bundles-r }}^{\text {hop }}
$$

$M_{\text {bundles-r }}$ is the message size (bits) of sold bundles and $\mathrm{N}_{\text {bundle- } \mathrm{r}}^{\text {hop }}$ is the number of hops the message $\mathrm{M}_{\text {bundles-r }}$ is transmitted to the winners in the $\mathrm{r}^{\text {th }}$ round of auctions. This cost also accumulates all the costs incurred for disseminating winner information for all auction rounds.

$\mathrm{C}_{\text {bid }}^{\text {session }}$ and $\mathrm{C}_{\text {winner }}^{\text {session }}$ are similarly computed based on Equations 8 and 9 respectively. But these auction costs occur only during the session time due to dynamics of node leave or join. We stress that we account for all the communication costs of running auctions due to the dynamics of node leave or join. Low $\mathrm{C}_{\text {total }}$ is desirable to reduce total communication overhead.

\section{NUMERICAL RESULTS AND ANALYSIS}

We validated our proposed protocol using SMPL [9], a discrete event driven simulator. Table 1 shows the default values used for the key design parameters.

TABLE I. DFAUlt VAlues For Key Design PARAMETERS

\begin{tabular}{|c|c|c|c|}
\hline Parameter & Value & Parameter & Value \\
\hline $\mathrm{N}_{\text {mission }}$ & 20 & $1 / \lambda$ & $1500 \mathrm{sec}$. \\
\hline $\mathrm{N}_{\text {preference }}^{\text {max }}$ & 3 & $1 / \mu$ & $500 \mathrm{sec}$. \\
\hline $\mathrm{N}_{\text {item }}^{\text {max }}$ & 3 & $\mathrm{~m}$ & $6 \times 6$ \\
\hline $\mathrm{N}$ & 100 & $\mathrm{~T}_{\text {next }}^{\text {auction }}$ & $10 \mathrm{sec}$. \\
\hline $\mathrm{T}_{\text {session }}$ & $10800 \mathrm{sec}$. & $1 / \delta_{\max }^{\text {workload }}$ & $10 \mathrm{sec}$. \\
\hline $\mathrm{N}_{\max }^{\text {mission }}$ & 20 & $\mathrm{R}$ & $250 \mathrm{~m}$ \\
\hline
\end{tabular}

The system is setup with the operational area based on square-shaped $6 \times 6$ sub-regions where each region has the same side of $250 \mathrm{~m}$, the wireless radio range $\mathrm{R}$. The required numbers of members per mission is generated based on uniform distribution in the range of $\left[1, \mathrm{~N}_{\max }^{\text {mission }}\right]$. The sum of the required numbers of members under all missions $\left(\sum_{\mathrm{k} \in \mathrm{G}} \mathrm{N}_{\mathrm{k}}^{\text {mission }}\right.$ where $\mathrm{N}_{\mathrm{k}}^{\text {mission }}$ indicates the required number of members for the execution of mission $\mathrm{k}$ ) varied between 200 and 230 based on 100 simulation runs. The workload of each mission is also based on uniform distribution with the range of $\left(0, \delta_{\max }^{\text {workload }}\right]$. We used a single-item auction with $\mathrm{N}_{\text {item }}^{\max }=1$ and a single-minded auction with $\mathrm{N}_{\text {preference }}^{\max }=1$ as baseline models for comparison with our proposed scheme. We study the effect of the proposed auction protocol on the three performance metrics (Section III.B) and provide physical interpretations of the simulation results.

\section{A. Total Communication Overhead}

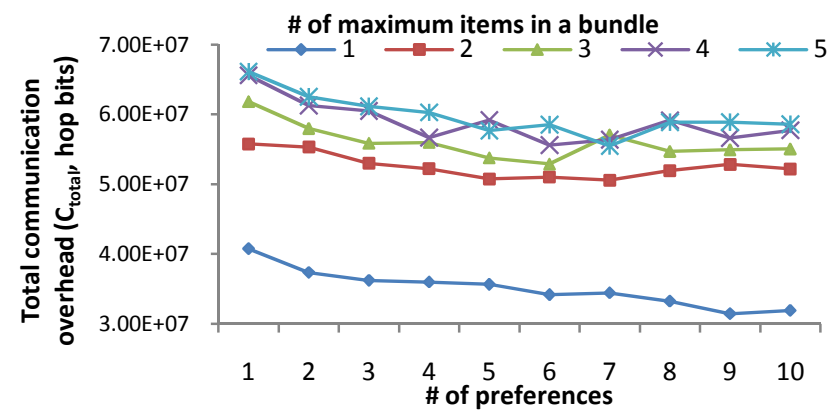

Fig. 2: The effect of the number of preferences on the total communication overhead with respect to the number of maximum items in a bundle.

First, Fig. 2 shows the effect of the number of preferences used $\left(\mathrm{N}_{\text {preference }}^{\max }\right)$ on the total communication overhead (Equation 6) incurred by the proposed resource allocation scheme as the number of maximum items $\left(\mathrm{N}_{\mathrm{item}}^{\max }\right)$ in a bundle varies. Overall, we observe that as $\mathrm{N}_{\text {preference }}^{\max }$ becomes larger, less communication cost is incurred due to fewer number of auction rounds introduced during network deployment. However, during the session, the effect of the number of auction rounds is trivial because many bundles are not available in the auction market and are only generated based on nodes leaving the network. Also as $\mathrm{N}_{\mathrm{item}}^{\max }$ becomes larger, higher communication cost is incurred because of the larger message sizes.

\section{B. Average Node Utilization}

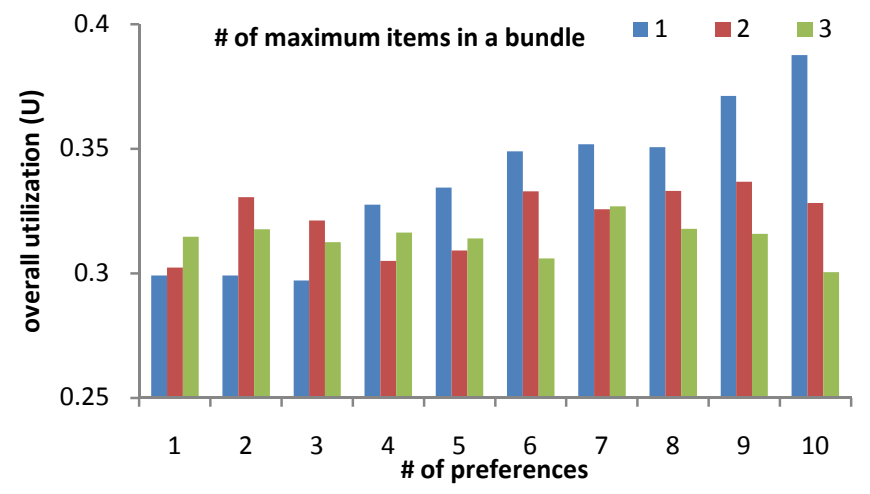

Fig. 3: The effect of the number of preferences on the average utilization of nodes with respect to the number of maximum items in a bundle.

In Fig. 3, we show how $\mathrm{N}_{\text {preference }}^{\max }$ affects the average node utilization (Equation 4) as $\mathrm{N}_{\text {item }}^{\max }$ varies. When $\mathrm{N}_{\text {item }}^{\max }$ is 
sufficiently small such as single-item auction with $\mathrm{N}_{\text {item }}^{\max }=1$, the overall node utilization (average busy time ratio) significantly increases as $\mathrm{N}_{\text {preference }}^{\max }$ increases. This is because bidding on multiple bundles with one mission item in each bundle provides nodes with more options to bid. But as $\mathrm{N}_{\mathrm{item}}^{\max }$ increases, we observe that no clear correlation exists between $\mathrm{N}_{\text {preference }}^{\max }$ and the overall node utilization (U). The reason is that a larger $\mathrm{N}_{\text {item }}^{\max }$ will decrease the number of generated bundles (fewer choices) which may result in some nodes not being assigned any bundles. Further, larger $\mathrm{N}_{\mathrm{item}}^{\mathrm{max}}$ may increase workload that may lower the score of each bundle to bidders, lowering the chance of a bid submission.

\section{Mission Completion Ratio}

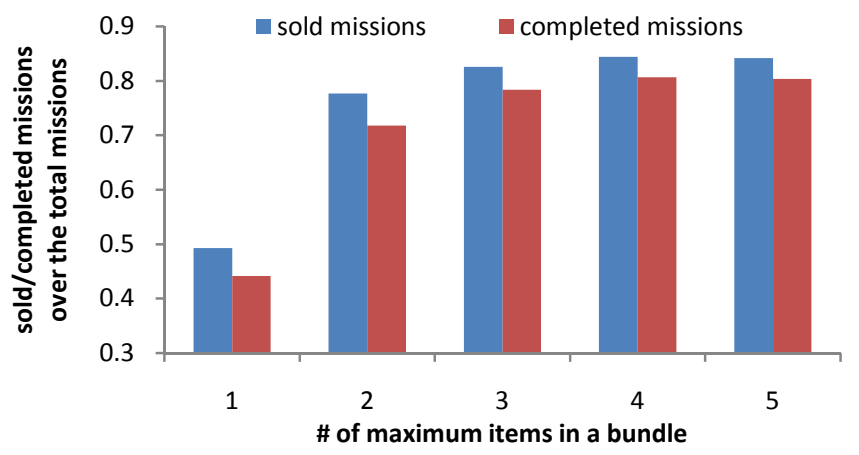

Fig. 4: The effect of the number of preferences on the mission completion ratio with respect to the number of maximum items in a bundle.

Fig. 4 shows the effect of $\mathrm{N}_{\text {item }}^{\max }$ on the mission completion ratio (Equation 5). We do not observe a significant effect of $\mathrm{N}_{\text {preference }}^{\max }$ on the mission completion ratio, and hence do not show the results here. In Fig. 4, we compared the ratios of the initially sold (assigned) missions and the completed missions over the total number of given mission tasks. The difference between the sold missions and completed missions is due to mission failures arising from dynamics of node leave or join. We clearly see higher mission completion ratio with larger $\mathrm{N}_{\text {item }}^{\max }$.

Overall we observed that smaller $\mathrm{N}_{\text {item }}^{\max }$ and larger $\mathrm{N}_{\text {preference }}^{\max }$ reduce the total communication overhead $\left(\mathrm{C}_{\text {total }}\right)$ and increase the average node utilization (U) while generating low mission completion ratio (M). That is, there exist tradeoffs between the communication overhead or average node utilization and mission completion ratio. We also noticed that there are no significant performance changes over the ranges of $\mathrm{N}_{\mathrm{item}}^{\max }>4$. Given constraints such as performance requirements in these three metrics, system designers can fine-tune the optimal setting of key design parameters such as $\mathrm{N}_{\text {item }}^{\max }$ or $\mathrm{N}_{\text {preference }}^{\max }$ for performance optimization.

\section{CONCLUSIONS}

We proposed a dynamic multiple mission assignment protocol where multiple missions arrive and should be completed at different times. Different from existing work, we studied a resource allocation problem where each node may be assigned to multiple missions during its lifetime while each mission may also be assigned to multiple nodes. We considered a MANET setting which leads to dynamic changes in available nodes; this in turn requires (partial) reallocation of idle nodes to the missions abandoned by leaving nodes. We used combinatorial auction theory as our tool for resource allocation and we showed how key design parameters can be adjusted to minimize communication overhead while maximizing node utilization and mission completion ratio.

Potential extensions of this work include: (1) investigating the sensitivity of performance metrics to other design parameters such as the session time $\left(\mathrm{T}_{\text {session }}\right)$, the total number of member nodes, the node join/leave rate, and the number of mission tasks; (2) comparing the performance of the proposed scheme with that of the global optimal scheme (for a small set of missions, given the complexity of computing the global solution); (3) adding other aspects of mission modeling such as importance or functional capability; and (4) modeling node leave based on an energy budget.

\section{REFERENCES}

[1] A. Ahmed, A. Patel, T. Brown, M.J. Ham, M. W. Jang, G. Agha, "Task assignment for a physical agent team via a dynamic forward/reverse auction mechanism," Int'l Conf. of Integration of Knowledge Intensive Multi-Agent Systems (KIMAS'05): Modeling, Evolutions and Engineering, pp. 311-317, 2005.

[2] B. An, V. Lesser, D. Irwin, and M. Zink, "Automated negotiation with decommitment for dynamic resource allocation in cloud computing," Proc. $9^{\text {th }}$ Int'l Conf. on Autonomous Agents and Multiagent Systems (AAMAS 2010), pp. 981-988, May 10-14, 2010.

[3] L. Blumrosen and N. Nisan, "Combinatorial auctions," Algorithmic Game Theory (Editor: N. Nisan et al.), Chapter 11, pp. 267-298, Campridge Universiry Press, 2007.

[4] J. Carlier and E. Pinson, "An algorithm for solving the job-shop problem," Journal Management Science, vol. 35, no. 2, Feb. 1989.

[5] J.H. Cho, I.R. Chen and D.C. Wang, "Performance optimization of region-based group key management in mobile ad hoc networks," Performance Evaluation, vol. 65, no. 5, pp 319-344, 2008.

[6] H. L. Choi, L. Brunet, and J. P. How, "Consensus-based decentralized auctions for robust task allocation," IEEE Transactions on Robotics, vol. 25, no. 4, pp. 912-926, Aug. 2009.

[7] M. P. Johnson, H. Rowaihy, D. Pizzocaro, A. Bar-Noy, S. Chalmers, T. F. La Porta, and A. Preece, "Sensor-mission assignment in constrained environments," IEEE Transactions on Parallel and Distributed Systems, vol. 21, no., pp. 1692-1705, 11, Nov. 2010.

[8] W.Y. Lin, G.Y. Lin, and H.Y. Wei,"Dynamic auction mechanism for cloud resource allocation," $10^{\text {th }}$ IEEE/ACM Int'l Con. on Cluster, Cloud and Grid Computing, pp. 591-592, 2010.

[9] M. H. MacDougall, Simulating Computer Systems, Computer Systems Series, The MIT Press, 1987.

[10] G. Mainland, D.C. Parkes, and M. Welsh, "Decentralized, adaptive resource allocation for sensor networks," Proc. $2^{\text {nd }}$ USENIX/ACM Symposium on Networked Systems Design and Implementation (NSDI 2005), vol. 2. pp. 315-328, May 2005.

[11] T. Mullen, V. Avasarala, and D. Hall, "Customer-driven sensor management,” IEEE Intelligent Systems, pp. 41-49, March/April 2006.

[12] C. Park, K.R. Pattipati, W. An, and D.L. Kleinman, "Quantifying the impact of information and communication structures via distributed auction algorithm," IEEE Int'l Conf. on Systems Man and Cybernetics (SMC), pp. 2200-2207, Oct. 10-13, 2010.

[13] K. Tei, H. Muto, Y. Fukazawa, and S. Honiden, "Introducing auction scheme to route allocation to prolong the lifetime of ad hoc networks," Int'l Conf. on Computational Intelligence for Modeling Control and Automation, 2006. 